\title{
Inertial effects in the nonlinear transient relaxation of rigid rodlike molecules in a strong dc electric field
}

\author{
Serguey V. Titov, ${ }^{1}$ Yuri P. Kalmykov, ${ }^{2, a)}$ and William T. Coffey ${ }^{3}$ \\ ${ }^{1}$ Institute of Radio Engineering and Electronics of the Russian Academy of Sciences, Vvedenskii Square 1, \\ Fryazino, Moscow Region 141190, Russian Federation \\ ${ }^{2}$ Laboratoire de Mathématiques, Physique et Systèmes, Université de Perpignan, 52 Avenue de Paul Alduy, \\ 66860 Perpignan Cedex, France \\ ${ }^{3}$ Department of Electronic and Electrical Engineering, Trinity College, Dublin 2, Ireland
}

(Received 11 July 2008; accepted 6 September 2008; published online 14 October 2008)

\begin{abstract}
A method of calculation of nonlinear transient responses of an assembly of noninteracting polar linear molecules due to sudden changes in a strong external dc electric field is presented. The infinite hierarchy of differential-recurrence relations for the decay functions describing the relaxation of the system is derived by averaging the underlying inertial Langevin equation. The solution of this hierarchy is obtained in terms of matrix continued fractions. The integral relaxation time and the spectrum of the electric polarization for various nonlinear transient responses (step-on, step-off, and rapidly rotating field) are calculated for typical values of the model parameters. The nonlinear transient responses exhibit pronounced nonlinear effects due to the strong dc field. Analytical equations for the quantities of interest are presented in the overdamped limit. Furthermore, the linear response relaxation function and linear dynamic susceptibility are obtained as a particular case of a general nonlinear theory. (C) 2008 American Institute of Physics. [DOI: 10.1063/1.2990748]
\end{abstract}

\section{INTRODUCTION}

An accurate method of solution of the inertial Langevin equation for particles undergoing Brownian movement (rotational or translational) subjected to strong external fields is essential in a variety of physical problems such as the nonlinear impedance of Josephson junctions, ${ }^{1}$ mobility of superionic conductors, ${ }^{2}$ nonlinear dielectric relaxation and dynamic Kerr effect of molecular liquids and nematic liquid crystals, ${ }^{3-8}$ and magnetic relaxation of ferrofluids. ${ }^{9,10}$ For example, inclusion of the inertia of the ions in superionic conductors is necessary in order to explain the conductivity spectrum at high frequencies. ${ }^{2}$ In the Josephson effect, the junction capacitance (playing the role of inertia) may substantially alter the response of the junction to a highfrequency alternating current. In the theory of dielectric and Kerr-effect relaxation, the inertia of molecules is neglected as usual at low frequencies. However, neglect of the molecular inertia in dielectric absorption leads to incorrect predictions (e.g., infinite absorption) of the behavior of dielectric spectra at high frequencies. ${ }^{4}$ Inertial effects may also appear in the spectra of the magnetic susceptibility of ferrofluids due to the physical rotation of the ferrofluid particles carrying the single domain magnetic particles. ${ }^{9,10}$

All the problems mentioned above require a nonlinear response theory with its inherent mathematical and physical complexity. The calculation of the nonlinear response, even for systems described by a single state variable, remains a difficult task as the response now depends on the precise nature of the stimulus. In other words, no unique response

\footnotetext{
${ }^{a)}$ Author to whom correspondence should be addressed. Electronic mail: kalmykov@univ-perp.fr.
}

function valid for all stimuli exists unlike in linear response. Existing nonlinear response theories of the above phenomena have usually been based on the Fokker-Planck equation for the appropriate probability density function $W{ }^{11}$ This equation can be solved by the method of separation of the variables. The separation procedure gives rise to a SturmLiouville problem. ${ }^{11}$ An alternative approach is to expand $W$ as a Fourier series in a complete set of appropriate orthogonal functions. The Fourier expansion yields an infinite hierarchy of recurrence equations for the statistical moments (the expectation values of the orthogonal functions), which may be solved by using scalar or matrix continued fraction methods. ${ }^{5,11}$ Yet another approach was developed by Coffey et al., ${ }^{5}$ who showed that the infinite hierarchy of recurrence equations for the moments can also be obtained by direct averaging of the underlying Langevin equation. The Langevin equation method and its applications to various linear and nonlinear response problems have been discussed in detail in Ref. 5.

In order to treat external field effects in nonlinear dielectric relaxation, the Langevin-Fokker-Planck model has been suitably generalized in a number of papers dealing with fixed axis rotators (see, for example, Refs. 5 and 12-15). Although the fixed axis rotator models reproduce the principal features of dielectric relaxation of an ensemble of dipolar molecules, they may be used solely for the qualitative evaluation of dielectric spectra because the rotators have only one degree of freedom and so do not allow one to quantitatively account for the effects of molecular inertia. Now inertial effects play a significant role in dielectric relaxation at high frequencies (e.g., the integral dipolar absorption and the shape of the spectra depend on them). Thus, a quantitative theory of nonlinear dielectric relaxation requires an analysis of molecular 
reorientations in space. In three dimensions, this problem is usually treated in the noninertial rotational diffusion limit (e.g., Refs. 7 and 16-21), thus the results are valid in the low-frequency region only, where $\omega \tau \leq 1$ ( $\tau$ is the orientational relaxation time).

The three-dimensional (3D) rotational motion in the presence of a strong electric field is, however, a difficult problem because the mathematics involved is much more complicated than that in the two-dimensional case. Here, we shall evaluate the nonlinear dielectric response of an assembly of linear molecules rotating in space, which is the simplest 3D model. We shall also consider a particular application of this model, namely, the transient nonlinear dielectric relaxation of a system of linear molecules rotating in space following a step change in a strong applied uniform electric field. Our approach relies on the solution of the recurrence equations for the statistical moments in terms of matrix continued fractions ${ }^{5,11}$ and may be regarded as an generalization of our results ${ }^{15}$ for the nonlinear response of an assembly of planar rotators.

\section{ROTATIONAL BROWNIAN MOTION AND TRANSIENT NONLINEAR RESPONSE}

We consider a system of noninteracting linear molecules (rotators) undergoing rotational Brownian motion in space in the presence of an external uniform dc electric field and suppose that the magnitude of the field is suddenly altered at time $t=0$ from $\mathbf{E}_{\mathrm{I}}$ to $\mathbf{E}_{\mathrm{II}}$. We are interested in the relaxation of the system of rotators starting from an equilibrium state I with the distribution function $W_{\mathrm{I}}(t \leq 0)$ to another equilibrium state II with the distribution function $W_{\text {II }}(t \rightarrow \infty)$. The distribution function in the equilibrium state $N=\mathrm{I}$, II is given by the Maxwell-Boltzmann distribution, viz.,

$$
W_{N}=Z_{N}^{-1} e^{-\eta^{2}\left(\dot{\vartheta}^{2}+\sin ^{2} \vartheta \dot{\varphi}^{2}\right)-V_{N}(\vartheta) / k T},
$$

where $Z_{N}$ is the partition function and the potential energy is given by

$$
V_{N}(\vartheta) /(k T)=-\xi_{N} \cos \vartheta
$$

Here $\vartheta$ and $\varphi$ are the polar and azimuthal angles, respectively, and

$$
\eta=\sqrt{\frac{I}{2 k T}}, \quad \xi_{N}=\frac{\mu E_{N}}{k T} .
$$

In Eqs. (1)-(3), I and $\mu$ are the moment of inertia and dipole moment of a rotator, respectively, $k$ is Boltzmann's constant, $T$ is the temperature, and $Z_{N}$ are the partition functions. This problem is truly nonlinear because the alteration in the magnitude of the strong external dc field is arbitrary with respect to the thermal energy $k T$. Here the internal field effects due to the interaction between the average moments and the Maxwell fields are not taken into account. Internal field effects in both linear and nonlinear responses always constitute a very difficult problem. However, these effects may be ignored for dilute solutions of polar molecules in first approximation.

Our goal is to evaluate the transient relaxation of the electric polarization $P(t)$ defined as

$$
P(t)=\mu N_{0}\langle\cos \vartheta\rangle(t)
$$

where $N_{0}$ is the concentration of dipoles and the angular brackets \langle\rangle$(t)$ mean the statistical average. Thus the dynamics of $P(t)$ can be described by the normalized relaxation function

$$
f(t)=\frac{\langle\cos \vartheta\rangle(t)-\langle\cos \vartheta\rangle_{\mathrm{II}}}{\langle\cos \vartheta\rangle_{\mathrm{I}}-\langle\cos \vartheta\rangle_{\mathrm{II}}} \quad(t>0) .
$$

Here, the angular brackets \langle\rangle$_{N}$ denote ensemble averages over the equilibrium distribution functions $W_{N}$, viz.,

$$
\langle\cos \vartheta\rangle_{N}=\operatorname{coth} \xi_{N}-1 / \xi_{N}
$$

Having determined $f(t)$ from Eq. (4), one may calculate the integral relaxation time $\tau$ defined as the area under the curve of the relaxation function, ${ }^{5}$

$$
\tau=\int_{0}^{\infty} f(t) d t
$$

In order to proceed, we now consider the 3D rotational diffusion of a linear polar molecule in an axially symmetrical external potential $V(\vartheta)$. In the molecular coordinate system oxyz fixed in the rotator, the angular velocity $\boldsymbol{\omega}$ and the angular momentum $\mathbf{L}$ of the rotator are defined $\mathrm{as}^{22}$

$$
\boldsymbol{\omega}=\left(\omega_{x}, \omega_{y}, \omega_{z}\right)=(\dot{\vartheta}, \dot{\varphi} \sin \vartheta, \dot{\varphi} \cos \vartheta)
$$

and

$$
\mathbf{L}=\left(I \omega_{x}, I \omega_{y}, 0\right)=(I \dot{\vartheta}, I \dot{\varphi} \sin \vartheta, 0)
$$

The rotational Brownian motion of the rotator is governed by the vector Euler-Langevin equation ${ }^{5}$

$$
\frac{d}{d t} \mathbf{L}(t)+\boldsymbol{\omega}(t) \times \mathbf{L}(t)+\zeta \boldsymbol{\omega}(t)=\mathbf{K}(t)+\boldsymbol{\lambda}(t),
$$

where $\mathbf{K}(t)$ is the torque due to the external potential $V$, and $\zeta \boldsymbol{\omega}(t)$ and $\boldsymbol{\lambda}(t)$ are the frictional and white noise torques due to the Brownian motion of the surroundings, respectively. The white noise torque has the following properties:

$$
\begin{aligned}
& \overline{\lambda_{j}(t)}=0, \quad \overline{\lambda_{j}(t) \lambda_{m}\left(t^{\prime}\right)}=2 \zeta k T \delta_{j, m} \delta\left(t-t^{\prime}\right) \\
& (j, m=x, y, z)
\end{aligned}
$$

where $\delta_{j, m}$ is Kronecker's delta and the overbar means the statistical average over an ensemble of rotators that all start at instant $t$ with the same sharp values of the angular velocity and the orientation. $\lambda_{j}(t)$ must also satisfy Isserlis's theorem for centered Gaussian random variables. ${ }^{5}$ We remark in passing that the white noise ansatz [Eq. (8)] should be regarded merely as a reasonable first approximation for the description of the dynamics of a molecule subjected to a strong field and at short time scales (where the inertial effects are vital) and that it may not be used to account for memory effects. If 
these are to be included, the random torque must have colored noise character. Equation (7) rewritten in terms of the components of the angular velocity in the moving axes becomes

$$
\begin{aligned}
& I \dot{\omega}_{x}(t)=I \omega_{y}^{2}(t) \cot \vartheta(t)-\zeta \omega_{x}(t)-\frac{\partial V}{\partial \vartheta}+\lambda_{x}(t), \\
& I \dot{\omega}_{y}(t)=-I \omega_{x}(t) \omega_{y}(t) \cot \vartheta(t)-\zeta \omega_{y}(t)+\lambda_{y}(t) .
\end{aligned}
$$

Equations (9) and (10) are stochastic differential equations. In order to treat them we shall use the Stratonovich interpretation $^{23}$ of such equations as that interpretation is the mathematical idealization of the dielectric relaxation process. ${ }^{5}$ Thus, it is unnecessary to transform Eqs. (9) and (10) to Itô equations (e.g., Ref. 11). Moreover, we can still apply conventional calculus. ${ }^{5,11}$

\section{RECURRENCE RELATIONS FOR STATISTICAL AVERAGES}

In order to generate differential-recurrence relations for the statistical moments, we now introduce, as in the free rotational Brownian motion (Ref. 5, Chap. 10), the functions

$$
f_{n}^{l, m}(t)=P_{l}^{m}[\cos \vartheta(t)] s_{n}^{m}\left[\omega_{x}(t), \omega_{y}(t)\right],
$$

where $P_{l}^{k}(z)$ are the associated Legendre functions ${ }^{24}$ and the orthogonal functions $s_{n}^{m}\left(\omega_{x}, \omega_{y}\right)$ are expressed as finite series of products of Hermite polynomials $H_{n}(z)$ in the components of the angular velocity, viz.,

$$
s_{n}^{2 m+M}\left(\omega_{x}, \omega_{y}\right)=\sum_{q=0}^{n} \frac{r_{2 m+M}(n, q)}{q !(n-q) !} H_{2 n-2 q+M}\left(\eta \omega_{x}\right) H_{2 q}\left(\eta \omega_{y}\right),
$$

where $M=0$ or 1 . Here the coefficients $r_{2 m+M}(n, q)$ are to be determined $^{5}$ from the following recurrence relations:

$$
\begin{aligned}
r_{2 m}(n, q)= & \left(n-q+\frac{1}{2}\right)\left(1-\frac{2 q+1}{2 m-1}\right) r_{2 m-1}(n, q) \\
& +(n-q) \frac{2 q+1}{2 m-1} r_{2 m-1}(n, q+1), \\
r_{2 m+1}(n, q) & =\left(1+\frac{q}{m}\right) r_{2 m}(n, q)-\frac{q}{m} r_{2 m}(n, q-1),
\end{aligned}
$$

with $r_{0}(n, q)=r_{1}(n, q)=1$. One has, for example, ${ }^{5} r_{2}(n, q)$ $=n-2 q, r_{3}(n, q)=n-4 q, r_{4}(n, q)=n(n-1)-8 q(n-q)$, etc.

The orthogonality properties of $s_{n}^{m}\left(\omega_{x}, \omega_{y}\right)$, viz.,

$$
\begin{aligned}
& \int_{-\infty}^{\infty} \int_{-\infty}^{\infty} s_{n}^{m}\left(\omega_{x}, \omega_{y}\right) s_{n^{\prime}}^{m^{\prime}}\left(\omega_{x}, \omega_{y}\right) e^{-\eta^{2}\left(\omega_{x}^{2}+\omega_{y}^{2}\right)} d \omega_{x} d \omega_{y} \\
& \quad \sim \delta_{n, n^{\prime}} \delta_{m, m^{\prime}}
\end{aligned}
$$

result from the orthogonality of the Hermite polynomials and Eqs. (13) and (14).

For a general axially symmetric potential function $V$ of the form

$$
\frac{V(\vartheta)}{k T}=\sum_{l=0}^{\infty} \nu_{l} P_{l}(\cos \vartheta),
$$

where $P_{l}(\cos \vartheta)$ are the Legendre polynomials, ${ }^{24}$ we can derive from Eqs. (9)-(12) equations for the statistical moments $\left\langle f_{n}^{l, k}\right\rangle(t)$ (the derivation is presented in Appendix A). Thus, we have

$$
\begin{aligned}
\eta \frac{d}{d t}\left\langle f_{n}^{l, 0}\right\rangle= & -2 n \beta^{\prime}\left\langle f_{n}^{l, 0}\right\rangle+\frac{1}{2}\left\langle f_{n}^{l, 1}\right\rangle+2\left\langle f_{n-1}^{l, 1}\right\rangle \\
& +\sum_{l_{1}=1}^{\infty} \nu_{l_{1}} \sum_{\substack{l_{2}=\left|l-l_{1}\right| \\
\Delta l_{2}=2}}^{l+l_{1}} \sqrt{\frac{l_{1}\left(l_{1}+1\right)}{l_{2}\left(l_{2}+1\right)}} C_{l, 0, l_{1}, 0}^{l_{2}, 0} C_{l, 0, l_{1}, 1}^{l_{2}, 1}\left\langle f_{n-1}^{l_{2}, 1}\right\rangle,
\end{aligned}
$$

and for $m \geq 1$

$$
\begin{aligned}
& \eta \frac{d}{d t}\left\langle f_{n}^{l, 2 m-M}\right\rangle=-(2 n+M) \beta^{\prime}\left\langle f_{n}^{l, 2 m-M}\right\rangle+\left\langle f_{n-1+M}^{l, 2 m+1-M}\right\rangle+\frac{1}{4}\left\langle f_{n+M}^{l, 2 m+1-M}\right\rangle-(l+2 m-M)(l-2 m+1+M) \\
& \times\left[\frac{n-m+1+M}{4}\left\langle f_{n+M}^{l, 2 m-1-M}\right\rangle+(n+m)\left\langle f_{n-1+M}^{l, 2 m-1-M}\right\rangle\right] \\
& +\sum_{l_{1}=1}^{\infty} \nu_{l_{1}} \sqrt{\frac{l_{1}\left(l_{1}+1\right)(l+2 m-M) !}{(l-2 m+M) !}}\left\{\sum_{\substack{l_{2}=\left|l-l_{1}\right| \\
\Delta l_{2}=2}}^{l+l_{1}} \sqrt{\frac{\left(l_{2}-2 m-1+M\right) !}{\left(l_{2}+2 m+1-M\right) !}} C_{l, 0, l_{1}, 0}^{l_{2}, 0} C_{l, 2 m-M, l_{1}, 1}^{l_{2}, 2 m+1-M}\left\langle f_{n-1+M}^{l_{2}, 2 m+1-M}\right\rangle\right. \\
& \left.-(n+m) \sum_{\substack{l_{2}=\left|l-l_{1}\right| \\
\Delta l_{2}=2}}^{l+l_{1}} \sqrt{\frac{\left(l_{2}-2 m+1+M\right) !}{\left(l_{2}+2 m-1-M\right) !}} C_{l, 0, l_{1}, 0}^{l_{2}, 0} C_{l, 2 m-M, l_{1},-1}^{l_{2}, 2 m-1-M}\left\langle f_{n-1+M}^{l_{2}, 2 m-1-M}\right\rangle\right\},
\end{aligned}
$$


where $C_{l, m, L, M}^{l^{\prime}, m^{\prime}}$ are the Clebsch-Gordan coefficients, $M=0$ or 1 , and $\beta^{\prime}=\zeta \eta / I$.

In the Brownian motion of a linear polar molecule in the presence of a dc electric field, all $\nu_{l}=0$ except $\nu_{1}=-\xi_{\mathrm{II}}$. Noting that at equilibrium the averages $\left\langle f_{n}^{l, m}\right\rangle_{\mathrm{II}}$ satisfy Eqs. (16) and (17), we can derive from Eqs. (16) and (17), the differential-recurrence relations for the relaxation functions $c_{n}^{l, m}(t)=\left\langle f_{n}^{l, m}\right\rangle(t)-\left\langle f_{n}^{l, m}\right\rangle_{\mathrm{II}}$, namely,

$$
\begin{aligned}
\eta \frac{d}{d t} c_{n}^{l, 0}= & -2 n \beta^{\prime} c_{n}^{l, 0}+\frac{1}{2} c_{n}^{l, 1}+2 c_{n-1}^{l, 1} \\
& +\frac{2 \xi_{\mathrm{II}}}{2 l+1}\left(c_{n-1}^{l-1,1}-c_{n-1}^{l+1,1}\right),
\end{aligned}
$$

$$
\begin{aligned}
\eta \frac{d}{d t} c_{n}^{l, 2 m-1}= & -(2 n+1) \beta^{\prime} c_{n}^{l, 2 m-1}+c_{n}^{l, 2 m}+\frac{1}{4} c_{n+1}^{l, 2 m} \\
& -(l+2 m-1)(l-2 m+2)\left[\frac{n-m+2}{4} c_{n+1}^{l, 2 m-2}\right. \\
& \left.+(n+m) c_{n}^{l, 2 m-2}\right]+\frac{\xi_{\mathrm{II}}}{2 l+1}\left\{c_{n}^{l-1,2 m}-c_{n}^{l+1,2 m}\right. \\
& -(n+m)\left[(l+2 m-2)(l+2 m-1) c_{n}^{l-1,2 m-2}\right. \\
& \left.\left.-(l-2 m+2)(l-2 m+3) c_{n}^{l+1,2 m-2}\right]\right\}
\end{aligned}
$$

$$
\begin{aligned}
\eta \frac{d}{d t} c_{n}^{l, 2 m}= & -2 n \beta^{\prime} c_{n}^{l, 2 m}+c_{n-1}^{l, 2 m+1}+\frac{1}{4} c_{n}^{l, 2 m+1}-(l+2 m)(l \\
& -2 m+1)\left[\frac{n-m+1}{4} c_{n}^{l, 2 m-1}+(n+m) c_{n-1}^{l, 2 m-1}\right] \\
& +\frac{\xi_{\mathrm{II}}}{2 l+1}\left\{c_{n-1}^{l-1,2 m+1}-c_{n-1}^{l+1,2 m+1}-(n+m)[(l\right. \\
& +2 m-1)(l+2 m) c_{n-1}^{l-1,2 m-1}-(l-2 m+1)(l \\
& \left.\left.-2 m+2) c_{n-1}^{l+1,2 m-1}\right]\right\} .
\end{aligned}
$$

Equations (18)-(20) can be solved by applying the one-sided Fourier transform and using matrix continued fractions (see Appendix B). Having determined the one-sided Fourier transform $\widetilde{c}_{0}^{1,0}(i \omega)=\int_{0}^{\infty} c_{0}^{1,0}(t) e^{-i \omega t} d t$, we can calculate the spectrum of the relaxation function $\widetilde{f}(\omega)$ of the transient nonlinear response, viz.,

$$
\tilde{f}(\omega)=\frac{\widetilde{c}_{0}^{1,0}(i \omega)}{c_{0}^{1,0}(0)},
$$

and the integral relaxation time $\tau$, viz.,

$$
\tau=\widetilde{f}(0)=\frac{\widetilde{c}_{0}^{1,0}(0)}{c_{0}^{1,0}(0)} .
$$

\section{RESULTS AND DISCUSSION}

The evolution of the relaxation time $\tau$ of the electric polarization as a function of the dimensionless damping (friction) parameter $\beta^{\prime}$ and the field parameters $\xi_{\mathrm{I}}$ and $\xi_{\mathrm{II}}$ (characterizing the strength of the dc field) is illustrated in

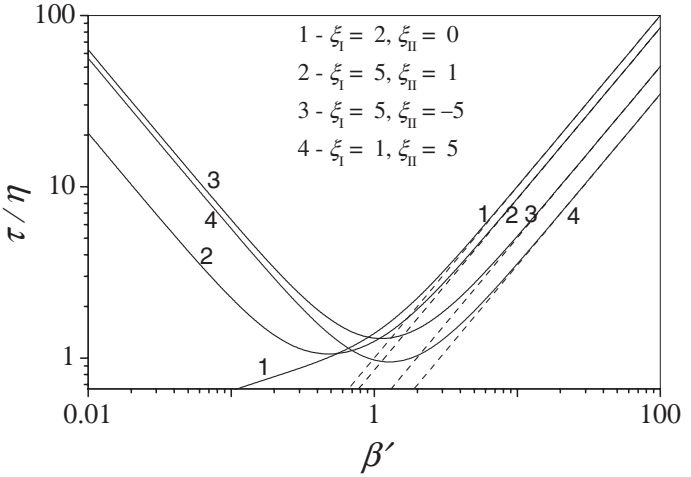

FIG. 1. Relaxation time $\tau / \eta$ for various transient responses as a function of the dimensionless damping parameter $\beta^{\prime}$. Solid lines: Calculations from Eq. (22); dashed lines: The overdamped limit [Eq. (24)].

Fig. 1 for various nonlinear transient responses, viz., step-on, step-off, and rapidly rotating field. Apparently in the overdamped limit $\left(\beta^{\prime} \gg 1\right)$, the relaxation time is directly proportional to the damping coefficient $\beta^{\prime}$. Such behavior can be readily understood if we recall that in the overdamped limit, the relaxational dynamics of linear rotators in the strong dc electric field is described by the single-variable FokkerPlanck equation (known in the case of separable and additive Hamiltonians as the Smoluchowski equation) for the axially symmetric probability density function $W(\vartheta, t)$ of the orientations of rotators, ${ }^{5}$

$$
\begin{aligned}
& 2 \tau_{D} \frac{\partial}{\partial t} W=\frac{1}{\sin \vartheta} \frac{\partial}{\partial \vartheta}\left[\sin \vartheta\left(\frac{\partial}{\partial \vartheta} W+\xi_{\text {II }} \sin \vartheta W\right)\right] \\
& (t>0),
\end{aligned}
$$

where $\tau_{D}=\eta \beta^{\prime}$ is the Debye relaxation time for isotropic noninertial rotational diffusion and the initial condition is

$$
W(\vartheta, 0)=W_{\mathrm{I}}^{0}(\cos \vartheta)=\frac{\xi_{\mathrm{I}} e^{\xi_{\mathrm{I}} \cos \vartheta}}{2 \sinh \xi_{\mathrm{I}}},
$$

$W_{\mathrm{I}}^{0}(\cos \vartheta)$ is the equilibrium distribution function [which is a stationary solution of Eq. (23)]. The relaxation time $\tau$ for nonlinear transient responses can be calculated for arbitrary values of $\xi_{\text {I }}$ and $\xi_{\text {II }}$ from the following equation: ${ }^{18}$

$$
\tau=\frac{2 \beta^{\prime} \eta}{\langle\cos \vartheta\rangle_{\mathrm{II}}-\langle\cos \vartheta\rangle_{\mathrm{I}}} \int_{-1}^{1} \frac{\Psi(z) \Phi(z)}{\left(1-z^{2}\right) W_{\mathrm{II}}^{0}(z)} d z,
$$

where $\Psi(z)=\int_{-1}^{z}\left(x-\langle\cos \vartheta\rangle_{\mathrm{II}}\right) W_{\mathrm{II}}^{0}(x) d x, \quad \Phi(z)=\int_{-1}^{z}\left(W_{\mathrm{II}}^{0}(x)\right.$ $\left.-W_{\mathrm{I}}^{0}(x)\right) d x$, and $W_{\mathrm{II}}^{0}(\cos \vartheta)=\xi_{\mathrm{II}} e^{\xi_{\mathrm{II}} \cos \vartheta} / 2 \sinh \xi_{\mathrm{II}}$. Thus, according to Eq. (24), the relaxation time is directly proportional to the damping coefficient $\beta^{\prime}$.

The real and imaginary parts of the one-sided Fourier transform of the relaxation function $f(t)$ are illustrated in Figs. 2-4. Here, it is clearly seen that in nonlinear transient responses, two processes appear in these spectra. The first is the single Debye-type relaxation process, which dominates the low-frequency part of the spectra and arising from the slow orientational relaxation mode of the dipole in the dc external electric field $\mathbf{E}_{\mathrm{II}}$. The characteristic frequency $\omega_{R}$ of this low-frequency mode corresponds to the inverse of the relaxation time $\tau$. This behavior implies that at long times 

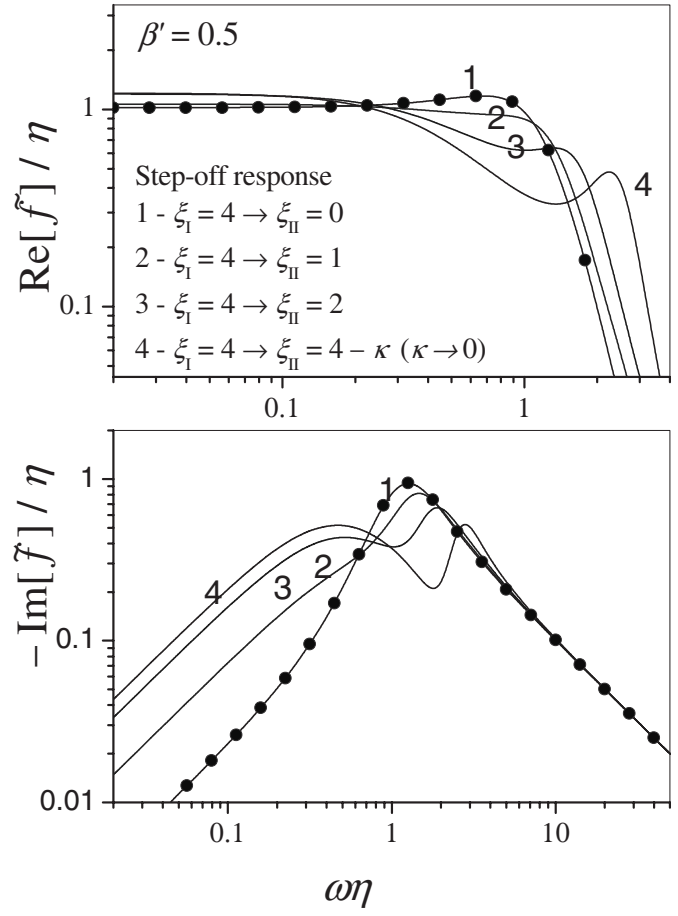

FIG. 2. Step-off transient response: $\operatorname{Re}[\widetilde{f}(\omega) / \eta]$ and $-\operatorname{Im}[\widetilde{f}(\omega) / \eta]$ as functions of $\omega \eta$ for $\xi_{\mathrm{I}}=4, \beta^{\prime}=0.5$, and various $\xi_{\mathrm{II}}$ : $\xi_{\mathrm{II}}=0$ (curve 1 ); $\xi_{\mathrm{II}}=1$ (curve $2) ; \xi_{I I}=2$ (curve 3$)$; and $-\xi_{I I}=4-\kappa(\kappa \rightarrow 0$, linear response; curve 4$)$. Filled circles: Calculations from Eq. (25).

$\left(t \omega_{R} \geq 1\right)$ the relaxation function $f(t)$ may be approximated by a single exponential, viz., $f(t) \approx \exp (-t / \tau)$, with one-sided Fourier transform spectrum

$$
\tilde{f}(\omega)=\frac{\tau}{1+i \omega \tau}
$$
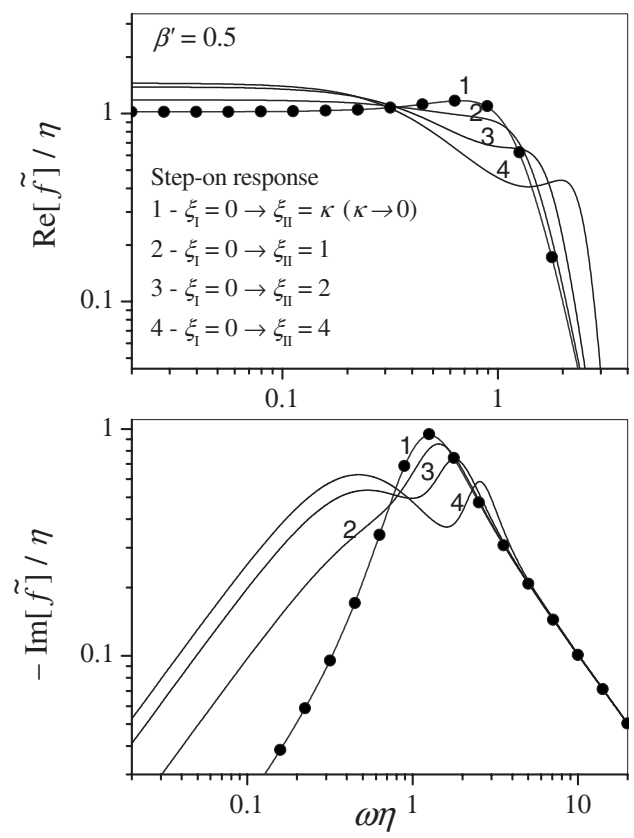

FIG. 3. Step-on transient response: $\operatorname{Re}[\widetilde{f}(\omega) / \eta]$ and $\operatorname{Im}[\widetilde{f}(\omega) / \eta]$ as functions of $\omega \eta$ for $\xi_{\mathrm{I}}=0, \beta^{\prime}=0.5$, and various $\xi_{\mathrm{II}}: \xi_{\mathrm{II}}=\kappa(\kappa \rightarrow 0$, linear response; curve 1); $\xi_{\mathrm{II}}=1$ (curve 2$) ; \xi_{\mathrm{II}}=2$ (curve 3 ); and $-\xi_{\mathrm{II}}=4$. Filled circles: Calculations from Eq. (25).
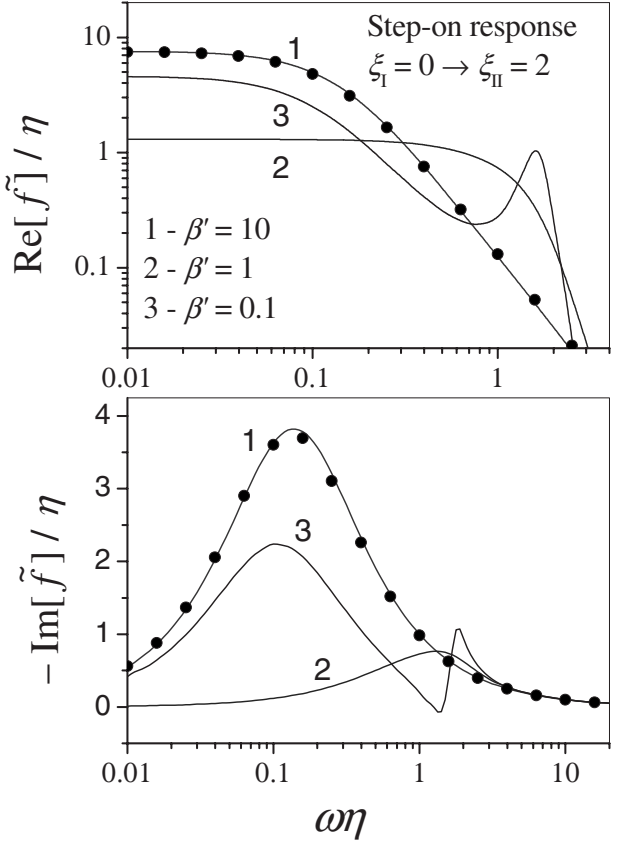

FIG. 4. Step-on transient response for $\xi_{\mathrm{I}}=0 \rightarrow \xi_{\mathrm{II}}=2: \operatorname{Re}[\widetilde{f}(\omega) / \eta]$ and $\operatorname{Im}[\tilde{f}(\omega) / \eta]$ as functions of $\omega \eta$ for various $\beta^{\prime}: \beta^{\prime}=10$ (curve 1); $\beta^{\prime}=1$ (curve 2); and $\beta^{\prime}=0.1$ (curve 3). Filled circles: The relaxation spectra calculated from Eq. (25) with $\tau / \eta \approx 0.475$ calculated from Eq. (24).

Here $\tau$ is the relaxation time evaluated from Eq. (22) (see Fig. 4). However, the second process embodies the most striking feature of the nonlinear response following the step alteration of the external field, namely, if the dc external electric field $\mathbf{E}_{\mathrm{II}}$ is sufficiently strong, then a pronounced resonantlike peak occurs in the high-frequency part of the spectrum due to the fast librations of the dipoles in the strong dc external electric field $\mathbf{E}_{\mathrm{II}}$ (see Figs. 2-4). This corresponds to the terahertz (far-infrared) band of frequencies. For $\xi_{\mathrm{II}} \gg 1$ and small damping $\left(\beta^{\prime} \approx 0\right)$, the characteristic frequency of librations $\omega_{L}$ can be estimated as $\omega_{L} \sim \eta^{-1} \sqrt{2 \xi_{\mathrm{II}}}$. On increasing $\beta^{\prime}$, although both processes still persist, the amplitude of the high-frequency process decreases progressively as one would intuitively expect. The resonantlike behavior of the nonlinear response spectrum corresponds in the time domain to the oscillations in the rise transient for large fields, which have been observed in computer simulations $^{25-27}$ and predicted already ${ }^{12}$ for the simple case of rotation about a fixed axis by solving the corresponding Fokker-Planck equation. The oscillatory behavior may be seen in Fig. 5 where the time domain response is plotted. Here, the calculation has been carried out numerically (using the fast Fourier transform algorithm) from the following equation:

$$
f(t)=\frac{1}{2 \pi} \operatorname{Re}\left\{\int_{0}^{\infty} e^{i \omega t} \tilde{f}(\omega) d \omega\right\}
$$

We may thus conclude that a sudden strong alteration in a 
uniform electric field applied to an assembly of noninteracting permanent dipoles will always give rise to pronounced resonance effects in the far-infrared region. We remark that for the step-off transient response with $\xi_{\mathrm{II}}=0$, the normalized relaxation function spectrum $\tilde{f}(\omega)$ coincides with that given by linear response theory and so does not exhibit resonantlike behavior. This spectrum was calculated by $\mathrm{Sack}^{28}$ and is given by the infinite continued fraction

$$
\tilde{f}(\omega)=\frac{\eta}{i \omega \eta+\frac{1}{\beta^{\prime}+i \omega \eta+\frac{1}{2 \beta^{\prime}+i \omega \eta+\frac{2}{3 \beta^{\prime}+i \omega \eta+\frac{2}{4 \beta^{\prime}+i \omega \eta+\cdots}}}}} .
$$

The above results indicate that strong field effects on orientational relaxation appear when the energy of the molecule in the field becomes comparable to or higher then the thermal energy. In actual experimental conditions, it is difficult to obtain values of the field parameter $\xi_{N} \geq 1$ for ordinary molecular liquids, where the molecules usually have a dipole moment $\mu=1-5$ D. However, many computer simulation data have been reported for such molecules (see, review $^{26}$ and references cited therein). In computer experiments, the values of $\xi_{N}$ were tested up to $\xi_{N} \sim 50$ and a variety of field effects were reported. ${ }^{27}$ In particular, initial oscillations of the first- and second-order orientational correlation functions (like those shown in Fig. 5) exist with these oscillations becoming more pronounced as the field strength increases before the relaxation functions settle into the familiar exponential decay. ${ }^{27}$ We remark as also noted in Ref. 27 that such a behavior cannot be interpreted at all using a noninertial rotational diffusion model. One should also note that a ferrofluid consisting of a colloidal suspension of blocked single domain ferromagnetic particles, whose size is approximately that of a true Brownian grain, may provide a better

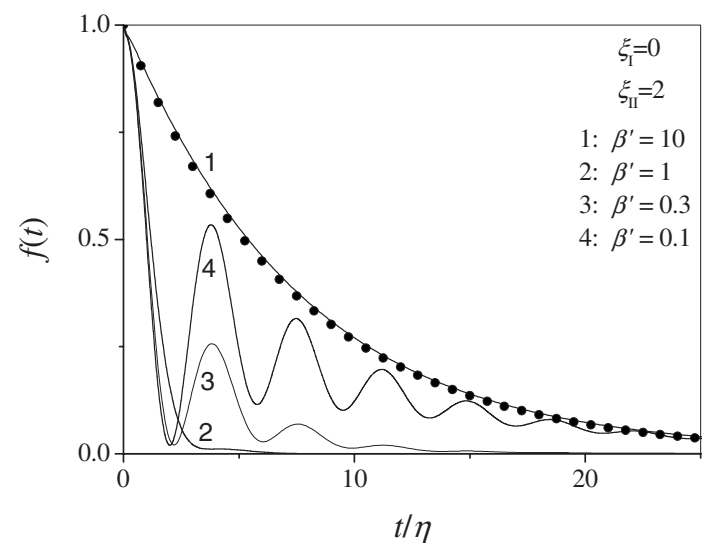

FIG. 5. Step-on transient response for $\xi_{\mathrm{I}}=1 \rightarrow \xi_{\mathrm{II}}=2: f(t)$ as a function of $t / \eta$ for various $\beta^{\prime}$. Curve 1: $\beta^{\prime}=10$ (overdamped limit); curve 2: $\beta^{\prime}=1$; and curve 3: $\beta^{\prime}=0.1$. Filled circles: The single exponential decay, viz., $\exp (-t / \tau)$ with the relaxation time $\tau / \eta \approx 7.157$ calculated from Eq. (24). system for the experimental verification of the high field induced oscillations rather than the minute electric dipoles characteristic of the Debye theory of dielectric relaxation. This is so because in a ferrofluid, due to the very large value of the magnetic moment $\left(10^{4}-10^{5} \mu_{B}\right)$ of a single domain particle, it is much easy to achieve a high value of the applied field parameter $\xi$ with a relatively small field rather than the very large fields, which must be used for electric dipoles with consequent risk of dielectric breakdown. ${ }^{5}$ In the ferrofluid, one would also expect the high-frequency induced oscillations to appear at radio frequencies rather than in the far-infrared region due to the very large moment of inertia of the ferrofluid particles.

The approach developed here also allows us to evaluate the linear response characteristics of the system of linear rotators to infinitesimally small changes in the strength of the strong dc field $\mathbf{E}_{\mathrm{I}}$, i.e., for $\xi_{\mathrm{II}}=\xi_{\mathrm{I}}-\kappa$, as $\kappa \rightarrow 0$ (see curve 4 in Fig. 2). The relaxation function $f(t)$ from Eq. (4) now coincides ${ }^{5}$ with the normalized longitudinal dipole equilibrium correlation function $C_{\|}(t)$, viz.,

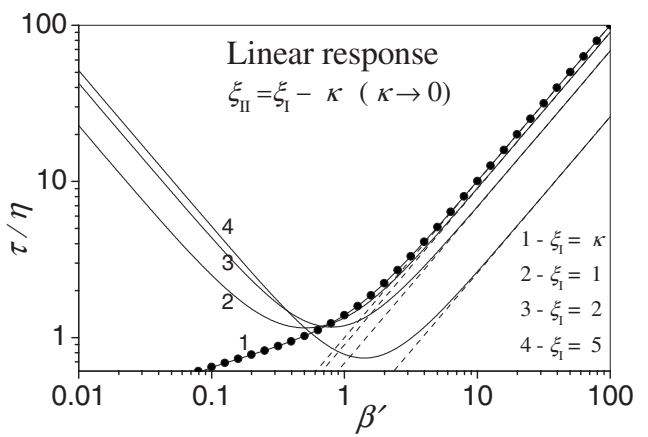

FIG. 6. Relaxation time $\tau / \eta$ for the linear response relaxation function in the presence of a dc field as a function of the dimensionless damping parameter $\beta^{\prime}$. Solid lines: Calculations from Eq. (22); dashed lines: The overdamped limit Eq. (24); and the filled circles: Eq. (28). 


$$
\lim _{\kappa \rightarrow 0} f(t)=C_{\|}(t)=\frac{\langle\cos \vartheta(0) \cos \vartheta(t)\rangle_{\mathrm{I}}-\langle\cos \vartheta(0)\rangle_{\mathrm{I}}^{2}}{\left\langle\cos ^{2} \vartheta(0)\right\rangle_{\mathrm{I}}-\langle\cos \vartheta(0)\rangle_{\mathrm{I}}^{2}}
$$

Having determined the one-sided Fourier transform of $C_{\|}(t)$, one can calculate the linear dynamic longitudinal susceptibility $^{5} \chi_{\|}(\omega)$

$$
\chi_{\|}(\omega)=\chi_{\|}(0)\left[1-i \omega \int_{0}^{\infty} C_{\|}(t) e^{-i \omega t} d t\right],
$$

where $\chi_{\|}(0)=\mu^{2} N_{0}\left[\left\langle\cos ^{2} \vartheta\right\rangle_{\mathrm{I}}-\langle\cos \vartheta\rangle_{\mathrm{I}}^{2}\right] / k T$ is the static susceptibility. This equation will yield the linear response to a small ac field $\mathbf{E}(t)=\mathbf{E} \exp (i \omega t)$ superimposed on a dc bias field $\mathbf{E}_{\mathrm{I}}$ of arbitrary strength since the polarization $P(t)$ is defined as

$$
P(t)=\mu N_{0}\left[\langle\cos \vartheta\rangle(t)-\langle\cos \vartheta\rangle_{\mathrm{I}}\right]=\chi_{\|}(\omega) E(t)
$$

The behavior of the relaxation time $\tau$ of the electric polarization for the linear response as a function of $\beta^{\prime}$ is illustrated in Fig. 6 for various values of the field parameters $\xi_{\mathrm{I}}$. Apparently, just as nonlinear response, the relaxation time in the overdamped limit $\left(\beta^{\prime} \gg 1\right)$ is directly proportional to the damping coefficient $\beta^{\prime}$. In the opposite low damping limit $\left(\beta^{\prime} \ll 1\right)$, the relaxation time is inversely proportional to the damping coefficient $\beta^{\prime}$. For $\xi_{\mathrm{I}} \rightarrow 0$, according to Eq. (26) the relaxation time $\tau$ is given by the continued fraction

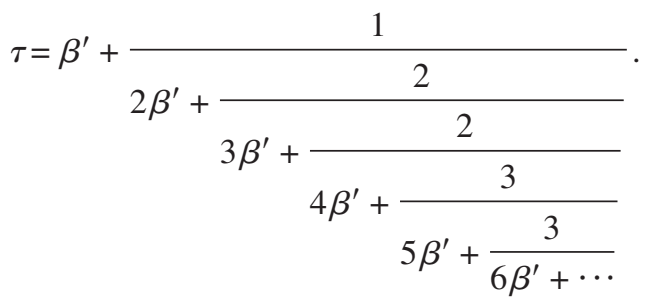

For linear response, the qualitative behavior of the susceptibility and relaxation time for the rotational Brownian motion under consideration is very similar to that of the extended rotational diffusion model of a linear molecule in a uniform dc electric field. ${ }^{29,30}$ We remark that the linear response for the simpler problem of the rotational Brownian motion of fixed axis rotators in a dc field has also been evaluated by Risken and Vollmer in Ref. 31.

\section{APPENDIX A: DERIVATION OF RECURRENCE EQUATIONS FOR STATISTICAL MOMENTS}

Recalling that conventional analysis applies to mathematical transformations of Stratonovich stochastic differential equations ${ }^{5}$ and noting Eqs. (9) and (10), we can form the following statistical averages of the associated Legendre $P_{l}^{m}[\cos \vartheta(t)]$ and $s_{n}^{m}\left[\omega_{x}(t), \omega_{y}(t)\right]$ functions:

$$
\begin{aligned}
s_{n}^{0}[ & \left.\omega_{x}(t), \omega_{y}(t)\right] \frac{d}{d t} P_{l}[\cos \vartheta(t)] \\
= & \frac{\omega_{x}(t) s_{n}^{0}\left[\omega_{x}(t), \omega_{y}(t)\right] P_{l}^{1}[\cos \vartheta(t)]}{n} \\
= & \frac{1}{2 \eta} P_{l}^{1} \sum_{q=0}^{n} \frac{1}{q !(n-q) !}\left[H_{2 n-2 q+1}^{x}\right. \\
& \left.+4(n-q) H_{2 n-2 q-1}^{x}\right] H_{2 q}^{y}=\frac{1}{2 \eta}\left(s_{n}^{1}+4 s_{n-1}^{1}\right) P_{l}^{1}
\end{aligned}
$$

and

$$
\begin{aligned}
P_{l}[\cos \vartheta(t)] \frac{d}{d t} s_{n}^{0}\left[\omega_{x}(t), \omega_{y}(t)\right] \\
=4 \eta P_{l} \sum_{q=0}^{n} \frac{1}{q !(n-q) !}\left[(n-q) H_{2 n-2 q-1}^{x} H_{2 q}^{y} \dot{\omega}_{x}\right. \\
\left.\quad+q H_{2 n-2 q}^{x} H_{2 q-1}^{y} \dot{\omega}_{y}\right]=-2 n \frac{\beta^{\prime}}{\eta} s_{n}^{0} P_{l}+g_{n-1}^{l, 0},
\end{aligned}
$$

where $H_{n}^{j}(t) \equiv H_{n}\left[\eta \omega_{j}(t)\right](j=x, y)$. Similarly, for $m \geq 1$ and $M=0$ or 1 , we have

$$
\begin{aligned}
s_{n}^{2 m+M}\left[\omega_{x}(t), \omega_{y}(t)\right] \frac{d}{d t} P_{l}^{2 m+M}[\cos \vartheta(t)] \\
=\omega_{x}(t) s_{n}^{2 m+M}\left[\omega_{x}(t), \omega_{y}(t)\right] \frac{\partial}{\partial \vartheta} P_{l}^{2 m+M}[\cos \vartheta(t)] \\
=\frac{1}{4 \eta}\left[P_{l}^{2 m+M+1}-(l+2 m+M)(l-2 m\right. \\
\left.\quad-M+1) P_{l}^{2 m+M-1}\right] \sum_{q=0}^{n} \frac{r_{2 m+M}(n, q)}{q !(n-q) !}\left[H_{2 n-2 q+M+1}^{x}\right. \\
\left.\quad+2(2 n-2 q+M) H_{2 n-2 q+M-1}^{x}\right] H_{2 q}^{y}
\end{aligned}
$$

and

$$
\begin{aligned}
\overline{P_{l}^{2 m+M}[\cos \vartheta(t)] \frac{d}{d t} s_{n}^{2 m+M}\left[\omega_{x}(t), \omega_{y}(t)\right]=} & -\frac{\beta^{\prime}}{\eta}(2 n+M) P_{l}^{2 m+M} s_{n}^{2 m+M}+g_{n+M-1}^{l, 2 m+M}-\frac{1}{4(2 m+M) \eta}\left[P_{l}^{2 m+M+1}+(l+2 m+M)\right. \\
& \left.\times(l-2 m-M+1) P_{l}^{2 m+M-1}\right] \sum_{q=0}^{n} \frac{(2 n-2 q+M) r_{2 m+M}(n, q)-2(n-q) r_{2 m+M}(n, q+1)}{q !(n-q) !} \\
& \times H_{2 n-2 q+M-1}^{x}\left[H_{2 q+2}^{y}+2(2 q+1) H_{2 q}^{y}\right] .
\end{aligned}
$$

Here 


$$
\begin{aligned}
& g_{n}^{l, 2 m-M}=\sum_{l_{1}=1}^{\infty} \nu_{l_{1}} \sqrt{\frac{l_{1}\left(l_{1}+1\right)(l+2 m-M) !}{(l-2 m+M) !}}\left\{\sum_{\substack{l_{2}=\left|l-l_{1}\right| \\
\Delta l_{2}=2}}^{l+l_{1}} \sqrt{\frac{\left(l_{2}-2 m-1+M\right) !}{\left(l_{2}+2 m+1-M\right) !}} C_{l, 0, l_{1}, 0}^{l_{2}, 0} C_{l, 2 m-M, l_{1}, 1}^{l_{2}, 2 m+1-M} \frac{f_{n-1+M}^{l_{2}, 2 m+1-M}}{l_{n-1}}\right. \\
& \left.-e_{n}^{2 m-M} \sum_{\substack{l_{2}=\left|l-l_{1}\right| \\
\Delta l_{2}=2}}^{l+l_{1}} \sqrt{\frac{\left(l_{2}-2 m+1+M\right) !}{\left(l_{2}+2 m-1-M\right) !}} C_{l, 0, l_{1}, 0}^{l_{2}, 0} C_{l, 2 m-M, l_{1},-1}^{l_{2}, 2 m-1, \frac{f_{n-1+M}, 2 m-1-M}{l_{2}}}\right\} \\
& e_{n}^{2 m}=\frac{2 r_{2 m}(n, q)-r_{2 m+1}(n-1, q)}{r_{2 m-1}(n-1, q)}=n+m, \\
& e_{n}^{2 m-1}=\frac{(2 n-2 q+1) r_{2 m-1}(n, q)-r_{2 m}(n, q)}{r_{2 m-2}(n, q)}=n+m .
\end{aligned}
$$

In order to obtain Eqs. (A1)-(A4), the following relations of the associated Legendre functions and the Hermite polynomials were used:

$$
\begin{aligned}
& \frac{d}{d x} H_{n}(x)=2 n H_{n-1}(x) \\
& H_{n+1}(x)=2 x H_{n}(x)-2 n H_{n-1}(x), \\
& \frac{\partial}{\partial \vartheta} P_{l}^{0}(\cos \vartheta)=P_{l}^{1}(\cos \vartheta) \\
& 2 \frac{\partial}{\partial \vartheta} P_{l}^{m}=P_{l}^{m+1}-(l+m)(l-m+1) P_{l}^{m-1},
\end{aligned}
$$

$2 m \cot \vartheta P_{l}^{m}=-P_{l}^{m+1}-(l+m)(l-m+1) P_{l}^{m-1}$,

$$
\begin{aligned}
& P_{l_{1}}^{1}(\cos \vartheta) P_{l}^{m}(\cos \vartheta)=\sqrt{\frac{l_{1}\left(l_{1}+1\right)(l+m) !}{(l-m) !}} \sum_{\substack{l_{2}=\left|l-l_{1}\right| \\
\Delta l_{2}=2}}^{l+l_{1}} \sqrt{\frac{\left[l_{2}-(m+1)\right] !}{\left[l_{2}+(m+1)\right] !} C_{l, 0, l_{1}, 0}^{l_{2}, 0} C_{l, m, l_{1}, 1}^{l_{2}, m+1} P_{l_{2}}^{m+1}(\cos \vartheta)} \\
& =\sqrt{\frac{l_{1}\left(l_{1}+1\right)(l+m) !}{(l-m) !}} \sum_{\substack{l_{2}=\left|l-l_{1}\right| \\
\Delta l_{2}=2}}^{l+l_{1}} \sqrt{\frac{\left[l_{2}-(m-1)\right] !}{\left[l_{2}+(m-1)\right] !}} C_{l, 0, l_{1}, 0}^{l_{2}, 0} C_{l, m, l_{1},-1}^{l_{2}, m-1} P_{l_{2}}^{m-1}(\cos \vartheta),
\end{aligned}
$$

and relations, which follow from Isserlis's theorem and which are given in Appendixes A-C of Ref. 5, viz.,

$$
\begin{gathered}
\overline{\lambda_{x}(t) F\left[\vartheta(t), \varphi(t), \omega_{y}(t)\right] H_{n}\left[\eta \omega_{x}(t)\right]} \\
=\frac{\zeta n}{\eta} F\left(\vartheta, \varphi, \omega_{y}\right) H_{n-1}\left(\eta \omega_{x}\right), \\
\overline{\lambda_{y}(t) G\left[\vartheta(t), \varphi(t), \omega_{x}(t)\right] H_{n}\left[\eta \omega_{y}(t)\right]} \\
=\frac{\zeta n}{\eta} G\left[\left(\vartheta, \varphi, \omega_{x}\right) H_{n-1}\left(\eta \omega_{y}\right)\right.
\end{gathered}
$$

( $F$ and $G$ are arbitrary functions). Noting that

$$
\overline{\frac{d}{d t} f_{n}^{l, m}(t)}=\overline{P_{l}^{m} \frac{d}{d t} s_{n}^{m}+s_{n}^{m} \frac{d}{d t} P_{l}^{m}}
$$

and combining Eqs. (A1)-(A4), we obtain the hierarchy of differential-recurrence equations

$$
\begin{aligned}
\eta \frac{d}{d t} \overline{f_{n}^{l, 0}}= & -2 n \beta^{\prime} \overline{f_{n}^{l, 0}}+\frac{1}{2} \overline{f_{n}^{l, 1}}+2 \overline{f_{n-1}^{l, 1}} \\
& +\sum_{l_{1}=1}^{\infty} \nu_{l_{1}} \sqrt{l_{1}\left(l_{1}+1\right)} \sum_{\substack{l_{2}=\left|l-l_{1}\right| \\
\Delta l_{2}=2}}^{l+l_{1}} \frac{C_{l, 0, l_{1}, 0}^{l_{2}, 0} C_{l, 0, l_{1}, 1}^{l_{2}, 1}}{\sqrt{l_{2}\left(l_{2}+1\right)}} f_{n-1}^{l_{2}, 1},
\end{aligned}
$$




$$
\begin{aligned}
\eta \frac{d}{d t} \overline{f_{n}^{l, 2 m-1}}= & -(2 n+1) \beta^{\prime} \overline{f_{n}^{l, 2 m-1}}+\overline{f_{n}^{l, 2 m}}+d_{n, 1}^{2 m-1} \overline{f_{n+1}^{l, 2 m}} \\
& -(l+2 m-1)(l-2 m+2)\left[d_{n,-1}^{2 m-1} \overline{f_{n+1}^{l, 2 m-2}}\right. \\
& \left.+d_{n, 0}^{2 m-1} \overline{f_{n}^{l, 2 m-2}}\right]+g_{n}^{l, 2 m-1}, \\
\eta \frac{d}{d t} \overline{f_{n}^{l, 2 m}}= & -2 n \beta^{\prime} \overline{f_{n}^{l, 2 m}}+\frac{1}{4} f_{n}^{l, 2 m+1}+d_{n, 1}^{2 m} \overline{f_{n-1}^{l, 2 m+1}}-(l+2 m) \\
& \times(l-2 m+1)\left[d_{n, 0}^{2 m} f_{n}^{l, 2 m-1}+d_{n,-1}^{2 m} f_{n-1}^{l, 2 m-1}\right] \\
& +g_{n}^{l, 2 m},
\end{aligned}
$$

where

$$
\begin{aligned}
d_{n, 0}^{2 m-1}= & \left(n-q+\frac{1}{2}\right)\left(1+\frac{2 q+1}{2 m-1}\right) \frac{r_{2 m-1}(n, q)}{r_{2 m-2}(n, q)} \\
& -\frac{(n-q)(2 q+1)}{2 m-1} \frac{r_{2 m-1}(n, q+1)}{r_{2 m-2}(n, q)}=n+m, \\
d_{n, 0}^{2 m}= & \frac{1}{4}\left[\left(1-\frac{q}{m}\right) \frac{r_{2 m}(n, q)}{r_{2 m-1}(n, q)}+\frac{q}{m} \frac{r_{2 m}(n, q-1)}{r_{2 m-1}(n, q)}\right] \\
= & \frac{n-m+1}{4}, \\
d_{n, \pm 1}^{2 m-1}= & \frac{(n-q+1)}{4}\left(1 \pm \frac{2 q}{2 m-1}\right) \frac{r_{2 m-1}(n, q)}{r_{2 m-1 \pm 1}(n+1, q)} \\
& \mp \frac{(2 n-2 q+3) q}{4(2 m-1)} \frac{r_{2 m-1}(n, q-1)}{r_{2 m-1 \pm 1}(n+1, q)} \\
= & \frac{1}{4}\left\{\begin{array}{c}
1 \\
n-m+2
\end{array}\right\},
\end{aligned}
$$

$$
\begin{aligned}
d_{n, \pm 1}^{2 m}= & \left(1 \mp \frac{2 q+1}{2 m}\right) \frac{r_{2 m}(n, q)}{r_{2 m \pm 1}(n-1, q)} \\
& \pm \frac{2 q+1}{2 m} \frac{r_{2 m}(n, q+1)}{r_{2 m \pm 1}(n-1, q)}=\left\{\begin{array}{c}
1 \\
n+m
\end{array}\right\} .
\end{aligned}
$$

Thus, averaging Eqs. (A5)-(A7) over the sharp initial conditions at the instant $t$, we obtain Eqs. (16) and (17).

\section{APPENDIX B: MATRIX CONTINUED FRACTION SOLUTION OF EQUATIONS (18)-(20)}

On introducing the column vectors

$$
\mathbf{C}_{n}(t)=\left(\begin{array}{c}
\mathbf{c}_{0}^{n}(t) \\
\mathbf{c}_{1}^{n}(t) \\
\vdots \\
\mathbf{c}_{n}^{n}(t) \\
\vdots \\
\mathbf{c}_{n}^{1}(t) \\
\mathbf{c}_{n}^{0}(t)
\end{array}\right)_{(n+1)(3 n / 2+1)} \quad, \quad \ldots \mathbf{c}_{n}^{l}=\left(\begin{array}{c}
c_{n}^{l, 0}(t) \\
c_{n}^{l, 1}(t) \\
\vdots \\
c_{n}^{l, l}(t)
\end{array}\right)_{l+1}
$$

the hierarchy of equations for the statistical moments $c_{n}^{l, m}(t)$ [Eqs. (18)-(20)] can now be transformed into the matrix three-term differential-recurrence equation

$$
\eta \frac{d}{d t} \mathbf{C}_{n}(t)=\mathbf{Q}_{n}^{-} \mathbf{C}_{n-1}(t)+\mathbf{Q}_{n} \mathbf{C}_{n}(t)+\mathbf{Q}_{n}^{+} \mathbf{C}_{n+1}(t)
$$

where $\mathbf{C}_{0}(t)=\mathbf{0}$ and the matrices $\mathbf{Q}_{n}^{-}, \mathbf{Q}_{n}$, and $\mathbf{Q}_{n}^{+}$are given by

$$
\begin{aligned}
& \mathbf{Q}_{n}=\left(\begin{array}{ccccccccc}
\mathbf{q}_{0, n} & \mathbf{q}_{0, n}^{+} & \mathbf{0} & \cdots & \mathbf{0} & \mathbf{0} & \mathbf{0} & \mathbf{0} & \mathbf{0} \\
\mathbf{q}_{1, n}^{-} & \mathbf{q}_{1, n} & \mathbf{q}_{1, n}^{+} & \ddots & \ddots & \mathbf{0} & \mathbf{0} & \mathbf{0} & \mathbf{0} \\
\mathbf{0} & \mathbf{q}_{2, n}^{-} & \ddots & \ddots & \mathbf{0} & \ddots & \mathbf{0} & \mathbf{0} & \mathbf{0} \\
\vdots & \ddots & \ddots & \mathbf{q}_{n-1, n} & \mathbf{q}_{n-1, n}^{+} & \mathbf{0} & \ddots & \mathbf{0} & \mathbf{0} \\
\ddots & \ddots & \mathbf{0} & \mathbf{q}_{n, n}^{-} & \mathbf{q}_{n, n} & \mathbf{p}_{n, n}^{-} & \mathbf{0} & \ddots & \mathbf{0} \\
\mathbf{0} & \ddots & \mathbf{0} & \mathbf{r}_{n, n-1}^{+} & \mathbf{p}_{n, n-1}^{+} & \mathbf{q}_{n, n-1} & \ddots & \ddots & \vdots \\
\mathbf{0} & \mathbf{0} & \ddots & \ddots & \mathbf{0} & \ddots & \ddots & \mathbf{p}_{n, 2}^{-} & \mathbf{0} \\
\mathbf{0} & \mathbf{0} & \mathbf{0} & \ddots & \ddots & \ddots & \mathbf{p}_{n, 1}^{+} & \mathbf{q}_{n, 1} & \mathbf{p}_{n, 1}^{-} \\
\mathbf{0} & \mathbf{0} & \mathbf{0} & \mathbf{0} & \mathbf{0} & \cdots & \mathbf{0} & \mathbf{p}_{n, 0}^{+} & \mathbf{q}_{n, 0}
\end{array}\right), \\
& \mathbf{Q}_{n}^{+}=\left(\begin{array}{cccccccccc}
\mathbf{p}_{0, n}^{+} & \mathbf{0} & \mathbf{0} & \mathbf{0} & \cdots & \mathbf{0} & \mathbf{0} & \mathbf{0} & \mathbf{0} & \mathbf{0} \\
\mathbf{r}_{1, n}^{+} & \mathbf{p}_{1, n}^{+} & \mathbf{0} & \mathbf{0} & \ddots & \ddots & \mathbf{0} & \mathbf{0} & \mathbf{0} & \mathbf{0} \\
\mathbf{0} & \mathbf{r}_{2, n}^{+} & \ddots & \ddots & \ddots & \ddots & \ddots & \mathbf{0} & \mathbf{0} & \mathbf{0} \\
\vdots & \ddots & \ddots & \ddots & \mathbf{0} & \mathbf{0} & \ddots & \ddots & \mathbf{0} & \mathbf{0} \\
\mathbf{0} & \ddots & \ddots & \mathbf{r}_{n, n}^{+} & \mathbf{p}_{n, n}^{+} & \mathbf{0} & \mathbf{q}_{n, n}^{+} & \ddots & \ddots & \mathbf{0} \\
\mathbf{0} & \mathbf{0} & \ddots & \ddots & \mathbf{0} & \mathbf{0} & \mathbf{0} & \ddots & \mathbf{0} & \vdots \\
\mathbf{0} & \mathbf{0} & \mathbf{0} & \ddots & \ddots & \ddots & \ddots & \ddots & \mathbf{q}_{n, 1}^{+} & \mathbf{0} \\
\mathbf{0} & \mathbf{0} & \mathbf{0} & \mathbf{0} & \cdots & \mathbf{0} & \mathbf{0} & \mathbf{0} & \mathbf{0} & \mathbf{q}_{n, 0}^{+}
\end{array}\right),
\end{aligned}
$$




$$
\mathbf{Q}_{n}^{-}=\left(\begin{array}{cccccccc}
\mathbf{p}_{0, n}^{-} & \mathbf{0} & \cdots & \mathbf{0} & \mathbf{0} & \mathbf{0} & \mathbf{0} & \mathbf{0} \\
\mathbf{r}_{1, n}^{-} & \mathbf{p}_{1, n}^{-} & \ddots & \ddots & \mathbf{0} & \mathbf{0} & \mathbf{0} & \mathbf{0} \\
\mathbf{0} & \mathbf{r}_{2, n}^{-} & \ddots & \ddots & \ddots & \mathbf{0} & \mathbf{0} & \mathbf{0} \\
\mathbf{0} & \ddots & \ddots & \ddots & \ddots & \ddots & \mathbf{0} & \mathbf{0} \\
\mathbf{0} & \ddots & \ddots & \ddots & \mathbf{p}_{n-1, n}^{-} & \ddots & \ddots & \mathbf{0} \\
\vdots & \ddots & \ddots & \mathbf{0} & \mathbf{r}_{n, n}^{-} & \mathbf{0} & \ddots & \vdots \\
\mathbf{0} & \ddots & \ddots & \mathbf{0} & \mathbf{q}_{n, n-1}^{-} & \ddots & \ddots & \mathbf{0} \\
\mathbf{0} & \mathbf{0} & \ddots & \ddots & \mathbf{r}_{n, n-2}^{+} & \ddots & \mathbf{r}_{n, 2}^{-} & \mathbf{0} \\
\mathbf{0} & \mathbf{0} & \mathbf{0} & \ddots & \ddots & \ddots & \mathbf{q}_{n, 1}^{-} & \mathbf{r}_{n, 1}^{-} \\
\mathbf{0} & \mathbf{0} & \mathbf{0} & \mathbf{0} & \cdots & \mathbf{0} & \mathbf{r}_{n, 0}^{+} & \mathbf{q}_{n, 0}^{-}
\end{array}\right)
$$

where $\mathbf{q}_{n, l}, \mathbf{q}_{n, l}^{ \pm}, \mathbf{r}_{n, l}^{ \pm}$, and $\mathbf{p}_{n, l}^{ \pm}$are submatrices with matrix elements defined as

$$
\begin{aligned}
& \underset{i, j=1,2, \ldots, l+1}{\left(\mathbf{q}_{n, l}\right)_{i j}}=\left\{\begin{array}{l}
-\delta_{i-1, j} \frac{1}{4}\left(n-\frac{i-3}{2}\right)(l+i-1)(l+2-i)-\delta_{i, j} 2 n \beta^{\prime}+\delta_{i+1, j} \frac{1}{4}\left(1+\delta_{i, 1}\right), \quad i=\text { odd } \\
-\delta_{i-1, j}(n+i / 2)(l+i-1)(l+2-i)-\delta_{i, j}(2 n+1) \beta^{\prime}+\delta_{i+1, j}, \quad i=\text { even, }
\end{array}\right. \\
& \underset{i, j=1,2, \ldots, l+1}{\left(\mathbf{q}_{n, l}^{+}\right)_{i j}}=\frac{1}{4}\left\{\begin{array}{l}
0, \quad i=\text { odd } \\
-\delta_{i-1, j}(n+2-i / 2)(l+i-1)(l+2-i)+\delta_{i+1, j}, \quad i=\text { even }
\end{array}\right. \\
& \left.\underset{i, j=1,2, \ldots,+1+1}{\left(\mathbf{q}_{n, l}^{-}\right.}\right)_{i j}=\left\{\begin{array}{l}
-\delta_{i-1, j}[n+(i-1) / 2](l+i-1)(l+2-i)+\delta_{i+1, j}\left(1+\delta_{i, 1}\right), \quad i=\text { odd } \\
0, \quad i=\text { even, }
\end{array}\right. \\
& \underset{i=1,2, \ldots l+1}{\left(\mathbf{p}_{,, l}^{+}\right)_{i j}}=\frac{\xi_{N}}{2 l+1}\left\{\begin{array}{l}
0, \quad i=\text { odd } \\
\delta_{i-1, j}(n+i / 2)(l+2-i)(l+3-i)-\delta_{i+1, j}, \quad i=\text { even, }
\end{array}\right. \\
& j=1,2, \ldots l+2 \\
& \underset{i=1,2, \ldots, l+1}{\left(\mathbf{p}_{n, l}^{-}\right)_{i j}}=-\frac{\xi_{N}}{2 l+1}\left\{\begin{array}{l}
0, \quad i=\text { odd } \\
\delta_{i-1, j}(n+i / 2)(l-2+i)(l-1+i)-\delta_{i+1, j}, \quad i=\text { even },
\end{array}\right. \\
& j=1,2, \ldots l \\
& \underset{i=1,2, \ldots, l+2}{\left(\mathbf{r}_{n, l}^{+}\right)_{i j}}=\frac{\xi_{N}}{2 l+1}\left\{\begin{array}{l}
\delta_{i-1, j}[n+(i-1) / 2](l+3-i)(l+2-i)-\delta_{i+1, j}\left(1+\delta_{i, 1}\right), \quad i=\text { odd } \\
0, \quad i=\text { even, }
\end{array}\right. \\
& j=1,2, \ldots l+1 \\
& \underset{i=1,2, \ldots l}{\left(\mathbf{r}_{n, l}^{-}\right)_{i j}}=-\frac{\xi_{N}}{2 l+1}\left\{\begin{array}{l}
\delta_{i-1, j}[n+(i-1) / 2](l-1+i)(l-2+i)-\delta_{i+1, j}\left(1+\delta_{i, 1}\right), \quad i=\text { odd } \\
0, \quad i=\text { even. }
\end{array}\right. \\
& j=1,2, \ldots l+1
\end{aligned}
$$

On taking the Laplace transform of Eq. (B1), we have the matrix three-term recurrence relation

$$
\left(\eta s \mathbf{I}_{n}-\mathbf{Q}_{n}\right) \widetilde{\mathbf{C}}_{n}(s)-\mathbf{Q}_{n}^{+} \widetilde{\mathbf{C}}_{n+1}(s)-\mathbf{Q}_{n}^{-} \widetilde{\mathbf{C}}_{n-1}(s)=\eta \mathbf{C}_{n}(0),
$$

where $\widetilde{\mathbf{C}}_{n}(s)=\int_{0}^{\infty} \mathbf{C}_{n}(t) e^{-s t} d t$ and $\mathbf{I}_{n}$ is the unit matrix. The exact solution of Eq. (B2) for the Laplace transform $\widetilde{\mathbf{C}}_{1}(s)$ is then given in terms of matrix continued fractions as ${ }^{5}$

$$
\widetilde{\mathbf{C}}_{1}(s)=\eta \boldsymbol{\Delta}_{1}(s)\left[\mathbf{C}_{1}(0)+\sum_{n=1}^{\infty}\left(\prod_{k=1}^{n} \mathbf{Q}_{k}^{+} \boldsymbol{\Delta}_{k+1}(s)\right) \mathbf{C}_{n+1}(0)\right],
$$

where 


$$
\boldsymbol{\Delta}_{n}(s)=\frac{\mathbf{I}_{n}}{\eta s \mathbf{I}_{n}-\mathbf{Q}_{n}-\mathbf{Q}_{n}^{+} \frac{\mathbf{I}_{n+1}}{\eta s \mathbf{I}_{n+1}-\mathbf{Q}_{n+1}-\mathbf{Q}_{n+1}^{+} \frac{\mathbf{I}_{n+2}}{\eta s \mathbf{I}_{n+2}-\mathbf{Q}_{n+2} \cdot} \mathbf{Q}_{n+2}^{-}} \mathbf{Q}_{n+1}^{-}}
$$

is the relevant matrix continued fraction (here the fraction lines denote matrix inversions).

The initial value vectors $\mathbf{C}_{n}(0)$ in Eq. (B3) are calculated in the following manner. We introduce the vector

$$
\mathbf{R}_{n}^{N}=\left(\begin{array}{c}
\left\langle f_{0}^{n, 0}\right\rangle_{N} \\
0 \\
\vdots \\
0
\end{array}\right)_{(n+1)(3 n / 2+1)}
$$

where

$$
\overline{\left\langle f_{0}^{n, 0}\right\rangle_{N}}=\frac{\int_{0}^{\pi} P_{n}(\cos \vartheta) e^{\xi_{N} \cos \vartheta} \sin \vartheta d \vartheta}{\int_{0}^{\pi} e^{\xi_{N} \cos \vartheta} \sin \vartheta d \vartheta}=\frac{I_{n+1 / 2}\left(\xi_{N}\right)}{I_{1 / 2}\left(\xi_{N}\right)}
$$

and $I_{n}(\xi)$ are the modified Bessel functions of the first kind. Here we have noted that $\left\langle\overline{f_{n}^{l, m}}\right\rangle_{N}=0$, for $n \neq 0$ and $m \neq 0$. Thus, we can write the initial value vector $\mathbf{C}_{n}(0)$ as $\mathbf{C}_{n}(0)$ $=\mathbf{R}_{n}^{\mathrm{I}}-\mathbf{R}_{n}^{\mathrm{II}}$. We note that for a particular case of the step-on transient response, namely, $\xi_{\mathrm{I}}=0, \xi_{\mathrm{II}} \neq 0$ (i.e., when a strong dc field is suddenly switched on), Eq. (B3) can be considerably simplified and is given by

$$
\widetilde{\mathbf{C}}_{1}(s)=\frac{1}{s}\left[\boldsymbol{\Delta}_{1}(s)-\boldsymbol{\Delta}_{1}(0)\right] \mathbf{Q}_{1}^{-}
$$

The matrix continued fraction solution we have obtained is easily computed.

\footnotetext{
${ }^{1}$ A. Barone and G. Paterno, Physics and Applications of the Josephson Effect (Wiley, New York, 1982).

${ }^{2}$ W. Dieterich, P. Fulde, and I. Peschel, Adv. Phys. 29, 527 (1980).

${ }^{3}$ W. T. Coffey, M. W. Evans, and P. Grigolini, Molecular Diffusion and Spectra (Wiley, New York, 1984) (Russian translation: Mir, Moscow, 1987).
}

${ }^{4}$ W. T. Coffey, Adv. Chem. Phys. 63, 69 (1985).

${ }^{5}$ W. T. Coffey, Yu. P. Kalmykov, and J. T. Waldron, The Langevin Equation, 2nd ed. (World Scientific, Singapore, 2004).

${ }^{6}$ L. M. Blinov, Electro-Optical and Magneto-Optical Properties of Liquid Crystals (Wiley, Chichester, 1983).

${ }^{7}$ H. Watanabe and A. Morita, Adv. Chem. Phys. 56, 255 (1984).

${ }^{8}$ S. Kielich, Molecular Nonlinear Optics (Science, Moscow, 1981).

${ }^{9}$ W. T. Coffey and Yu. P. Kalmykov, J. Magn. Magn. Mater. 164, 133 (1996).

${ }^{10}$ C. Scherer and H. G. Matuttis, Phys. Rev. E 63, 011504 (2001).

${ }^{11}$ H. Risken, The Fokker-Planck Equation, 2nd ed. (Springer, Berlin, 1989).

${ }^{12}$ W. T. Coffey, C. Rybarcsh, and W. Schröer, Chem. Phys. Lett. 99, 31 (1983).

${ }^{13}$ J. Cislo and M. Dudek, J. Chem. Phys. 100, 1567 (1994).

${ }^{14}$ W. T. Coffey, J. Chem. Phys. 93, 724 (1990).

${ }^{15}$ W. T. Coffey, Yu. P. Kalmykov, and S. V. Titov, J. Chem. Phys. 115, 9895 (2001)

${ }^{16}$ Yu. L. Raikher, V. I. Stepanov, and S. V. Burylov, J. Colloid Interface Sci. 144, 308 (1991).

${ }^{17}$ Yu. P. Kalmykov and S. V. Titov, Radiotekh. Elektron. 42, 578 (1997) [J. Commun. Technol. Electron. 42, 531 (1997)].

${ }^{18}$ Yu. P. Kalmykov, J. L. Déjardin, and W. T. Coffey, Phys. Rev. E 55, 2509 (1997).

${ }^{19}$ R. Ullman, J. Chem. Phys. 56, 1869 (1972).

${ }^{20}$ B. U. Felderhof and R. B. Jones, J. Chem. Phys. 115, 4444 (2001); 115, 7852 (2001).

${ }^{21}$ B. U. Felderhof, Phys. Rev. E 66, 051503 (2002).

${ }^{22}$ J. R. McConnell, Rotational Brownian Motion and Dielectric Theory (Academic, London, 1980).

${ }^{23}$ R. L. Stratonovich, Conditional Markov Processes and Their Application to the Theory of Optimal Control (Elsevier, New York, 1968).

${ }^{24}$ Handbook of Mathematical Functions, edited by M. Abramowitz and I. Stegun (Dover, New York, 1964).

${ }^{25}$ M. W. Evans, J. Chem. Phys. 77, 4632 (1982).

${ }^{26}$ M. W. Evans, Adv. Chem. Phys. 62, 183 (1985).

${ }^{27}$ A. Vegiri, J. Mol. Liq. 110, 155 (2004).

${ }^{28}$ R. A. Sack, Proc. Phys. Soc. London, Sect. B 70, 414 (1957).

${ }^{29}$ Yu. P. Kalmykov, Izv. Vyssh. Uchebn. Zaved., Radiofiz. 32, 1113 (1989) [ Radiophys. Quantum Electron. 32, 826 (1990)]; Yu. P. Kalmykov, Phys. Rev. A 45, 7184 (1992).

${ }^{30}$ S. V. Titov, Yu. P. Kalmykov, and W. T. Coffey, J. Chem. Phys. 118, 209 (2003).

${ }^{31}$ H. Risken and H. D. Vollmer, Mol. Phys. 46, 555 (1982). 